in vivo $35: 2975-2979$ (2021)

doi:10.21873/invivo.12592

\title{
Superior Mesenteric Vein Resection Followed by Porto-Jejunal Anastomosis During Pancreatoduodenectomy for Borderline Resectable Pancreatic Cancer - A Case Report and Literature Review
}

\author{
NICOLAE BACALBASA ${ }^{1,2}$, IRINA BALESCU ${ }^{3}$, VALENTIN VARLAS $^{2,4}$, MIHAI DIMITRIU $^{2,5}$, \\ CRISTIAN BALALAU ${ }^{6,7}$, FLORENTINA FURTUNESCU ${ }^{8}$, FLORENTINA GHERGHICEANU $^{9}$, \\ DANIEL RADAVOI ${ }^{10,11}$, CAMELIA DIACONU ${ }^{12,13}$, OVIDIU STIRU ${ }^{14,15}$, \\ CORNEL SAVU ${ }^{16,17}$, VLADISLAV BRASOVEANU ${ }^{1}$ and IOAN CORDOS ${ }^{16,17}$ \\ ${ }^{1}$ Department of Visceral Surgery, Center of Excellence in Translational \\ Medicine "Fundeni" Clinical Institute, Bucharest, Romania; \\ ${ }^{2}$ Department of Obstetrics and Gynecology, Carol Davila University \\ of Medicine and Pharmacy, Bucharest, Romania; \\ ${ }^{3}$ Department of Surgery "Ponderas" Academic Hospital, Bucharest, Romania; \\ ${ }^{4}$ Department of Obstetrics and Gynecology, “Filantropia” Hospital, Bucharest, Romania; \\ ${ }^{5}$ Department of Obstetrics and Gynecology, “St. Pantelimon” Emergency Hospital, Bucharest, Romania; \\ ${ }^{6}$ Department of Surgery, Carol Davila University of Medicine and Pharmacy, Bucharest, Romania; \\ ${ }^{7}$ Department of Surgery, "St. Pantelimon" Emergency Hospital, Bucharest, Romania; \\ ${ }^{8}$ Department of Public Health and Management University of Medicine \\ and Pharmacy "Carol Davila", Bucharest, Romania; \\ ${ }^{9}$ Department of Marketing and Medical Technology, "Carol Davila" \\ University of Medicine and Pharmacy, Bucharest, Romania; \\ ${ }^{10}$ Department of Urology, 'Prof. Dr. Th. Burghele' Clinical Hospital, Bucharest, Romania; \\ ${ }^{11}$ Department of Urology, "Carol Davila” University of Medicine and Pharmacy, Bucharest, Romania; \\ ${ }^{12}$ Department of Internal Medicine, "Carol Davila” University of Medicine and Pharmacy, Bucharest, Romania; \\ ${ }^{13}$ Department of Internal Medicine, Clinical Emergency Hospital of Bucharest, Bucharest, Romania; \\ ${ }^{14}$ Emergency Institute for Cardiovascular Diseases Prof. Dr. C.C. Iliescu, Bucharest, Romania; \\ ${ }^{15}$ Department of Cardio-Thoracic Pathology, "Carol Davila" \\ University of Medicine and Pharmacy, Bucharest, Romania; \\ ${ }^{16}$ Department of Thoracic Surgery, "Marius Nasta" National Institute of Pneumology, Bucharest, Romania; \\ ${ }^{17}$ Department of Thoracic Surgery, “Carol Davila” University of Medicine and Pharmacy, Bucharest, Romania
}

\begin{abstract}
Background/Aim: Pancreatic cancer represents the most lethal abdominal malignancy, the only chance for achieving an improvement in terms of survival being
\end{abstract}

This article is freely accessible online.

Correspondence to: Nicolae Bacalbasa, "Carol Davila" University of Medicine and Pharmacy, 37 Dionisie Lupu Street, 020021 Bucharest, Romania. Tel: +40 723540426, e-mail: nicolae_bacalbasa@yahoo.ro

Key Words: Borderline resectable pancreatic cancer, superior mesenteric vein, portal vein, invasion, reconstruction. represented by radical surgery. Although it has been considered that venous invasion represents a contraindication for resection, recently it has been demonstrated that in regards to overall survival after radical resection, it is similar to the one reported after standard pancreatoduodenectomy. Case Report: A 53year-old patient with no significant medical past was diagnosed with a borderline resectable pancreatic adenocarcinoma invading the superior mesenteric vein. The patient was submitted to pancreatoduodenectomy en bloc with superior mesenteric vein resection; the two jejunal veins were further anastomosed to the remnant portal vein. The postoperative outcome was favorable; the patient was discharged in the $10^{\text {th }}$ postoperative day. Conclusion: Although technically more 
demanding, pancreatoduodenectomy en bloc with superior mesenteric vein resection and jejunal portal anastomosis is feasible and might offer a chance for long-term survival in borderline pancreatic head carcinoma invading the superior mesenteric vein.

Pancreatic cancer remains the most aggressive abdominal malignancy, being associated with the poorest rates of longterm survival, especially in the absence of radical intent treatment (1). Therefore, while in cases in which curative surgery is not feasible the five-year survival rate is rather null, in cases in which radical resection is feasible the five-year survival rate reaches $25 \%$ (2-4). However, the close anatomic relationship between the pancreatic head and the portomesenteric system leads to the apparition of venous invasion in up to $30 \%$ of patients diagnosed with pancreatic head carcinoma (5). Although initially it has been thought that venous invasion is considered as the sign of a biologically very aggressive tumor, more recent studies demonstrated that the anatomical proximity of the entities and not the biological aggressiveness of the tumor is responsible for local invasion (6-9). In this respect, cases presenting venous invasion have been classified as borderline resectable ones and have been submitted to surgery whenever the biological and clinical status of the patient allows it (6-8). Meanwhile, improvement of the surgical techniques and of the postoperative care, and creation of tertiary centers of pancreatic surgery leaded to the significant increase in the rates of long-term survival in such cases, while the rates of perioperative complications significantly decreased $(3,4,9)$. Therefore, venous resection and reconstruction has become part of pancreatic surgery for borderline resectable pancreatic head carcinomas, and the long-term outcomes demonstrate similar rates of survival when compared to those reported after standard pancreatoduodenectomy (5-9). According to the level and extent of invasion, multiple techniques of resection and reconstruction have been proposed. Therefore, while in cases in which tangential invasion of the portal vein is present a lateral resection followed by suture can be performed, in cases in which circumferential invasion is present more extended vascular resections are needed. The reconstruction of the venous axis can be performed by an endto-end anastomosis in cases where a short segment is resected or by graft interposition if the length of the resected segment is higher (10). The aim of this study was to present the case of a patient presenting a borderline pancreatic head adenocarcinoma with superior mesenteric vein invasion in which resection followed by mesenterico-jejunal anastomosis was performed.

\section{Case Report}

After obtaining the approval of the Ethics Committee (no $11 / 2021$ ), data of the patient were presented in the current paper.
A 53-year-old patient with no significant medical antecedents self-presented for diffuse abdominal pain and weight loss of $14 \mathrm{~kg}$ in the last six months. The abdominal ultrasound revealed the presence of a $3 / 2 \mathrm{~cm}$ hypoechogenic mass developed at the level of the pancreatic head; meanwhile, no other pathological aspects were found. The biological tests revealed increased serum levels of cancer antigen 19-9 (CA 19-9) of $721 \mathrm{U} / \mathrm{ml}^{3}$ in association with slightly increased serum levels of gamaglutamiltranspeptidase (GGT) (measuring 198U/1), and of alkaline phosphatase (ALKP) (measuring $328 \mathrm{U} / 1$ ). The serum levels of transaminases, bilirubin, and lipase were normal. The patient was further submitted to a computed tomography, which demonstrated the presence of a $3.5 / 3.1 / 2.8 \mathrm{~cm}$ tumoral mass at the level of the pancreatic head invading the superior mesenteric vein and causing significant dilatation of the first jejunal veins; no signs of arterial invasion or distant metastases were encountered. An endoscopic ultrasound was therefore performed in order to confirm the local extension of the disease and to retrieve a biopsy. The histopathological studies confirmed the presence of a well differentiated pancreatic adenocarcinoma. Due to the good general and biological status of the patient, the final decision was to be submitted to surgery followed by adjuvant chemotherapy. Intraoperatively, the local invasion at the level of the superior mesenteric vein was confirmed with no other signs of local or distant lesions, thus the patient was submitted to pancreatoduodenectomy en bloc with superior mesenteric vein resection and partially with portal vein resection. The venous continuity was re-established by direct anastomosis between the remaining portal vein and distally with the first two jejunal veins (which were significantly dilated due to the presence of tumoral invasion of the upper segments) (Figures 1 and 2). Intraoperatively the estimated blood loss was 800 $\mathrm{ml}$ and the length of the surgical procedure was 450 minutes. Postoperatively, the patient received low molecular heparin for the next three weeks and was switched to oral anticoagulant for the next six months. The Doppler ultrasound revealed the absence of any signs of venous thrombosis at two days, one week, and six weeks follow up. The histopathological studies confirmed the presence of a well differentiated pancreatic adenocarcinoma with venous invasion affecting all the layers of the vascular structure.

\section{Discussion}

Initially performed by Moore et al. in 1951 and described as "regional pancreatectomy" by Fortner in $1973(11,12)$, venous resection during pancreatoduodenectomy has been considered as a too aggressive procedure in which the risks of perioperative complications surpass the benefits (13); however, improvement in the field of surgery and postoperative care as well as the development of tertiary 


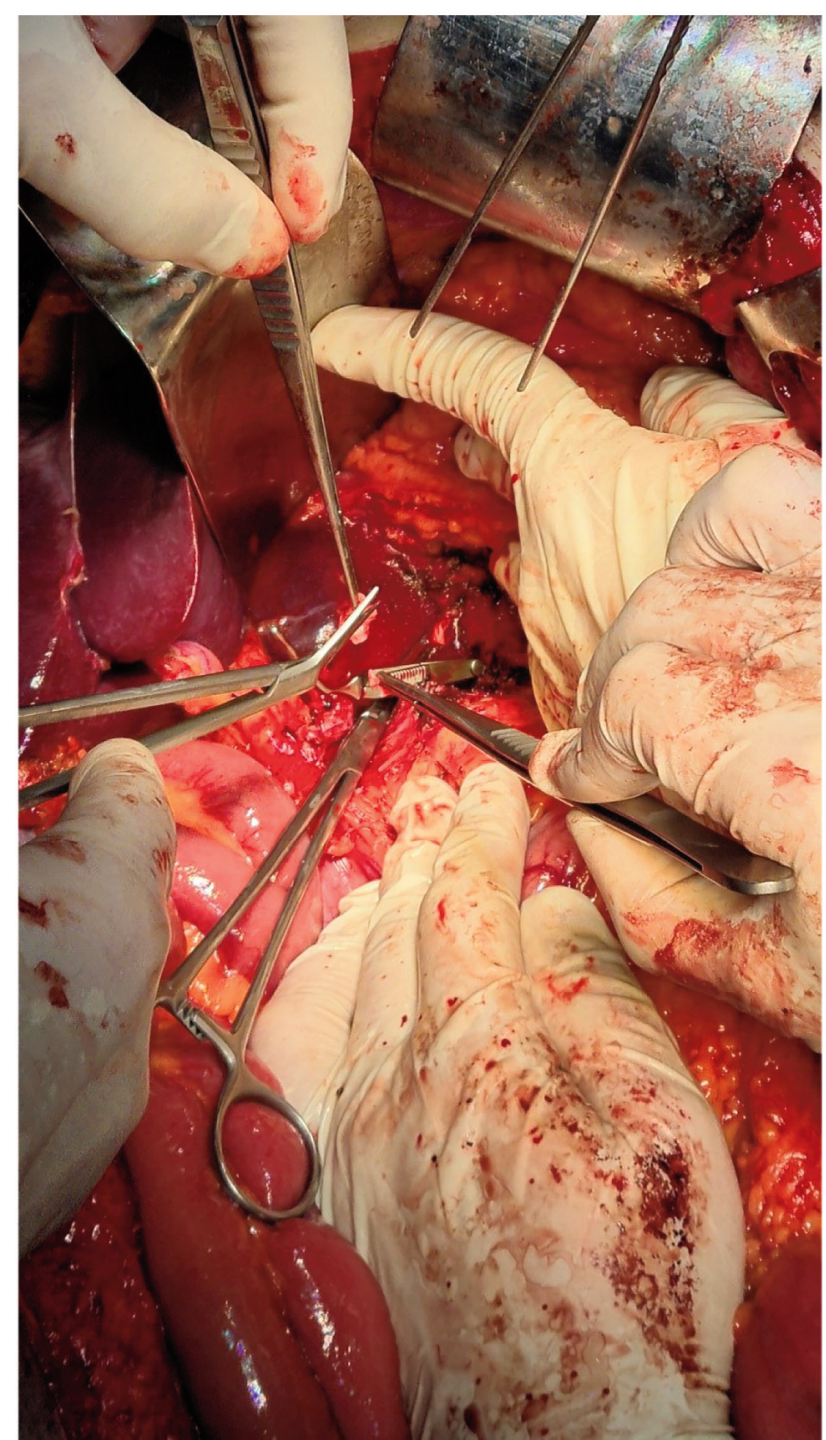

Figure 1. Preparing the portal vein for anastomosis.

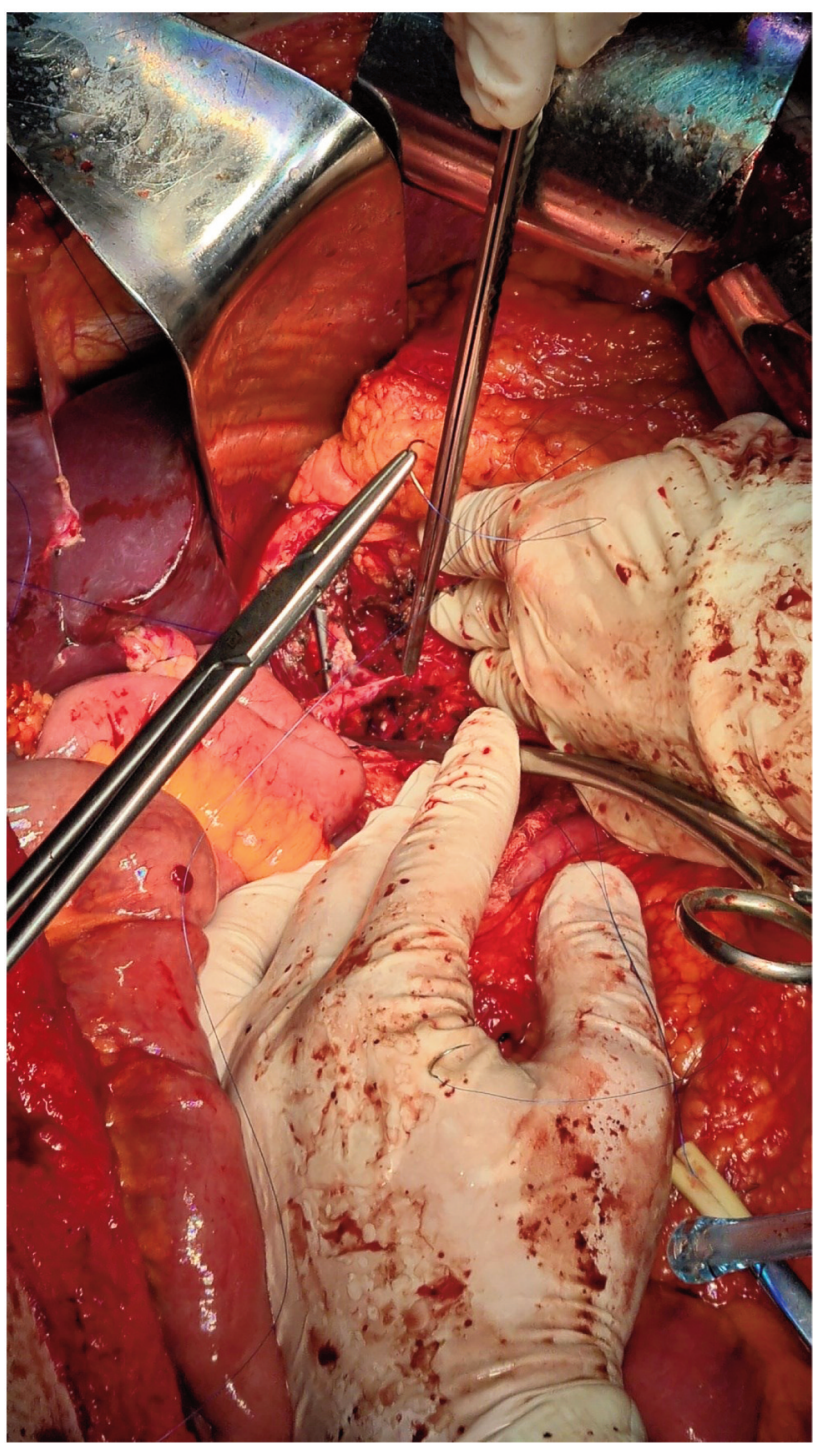

Figure 2. Intraoperative aspect: anastomosis between the remnant portal vein and the dilated jejunal veins.

venous suture, type 2 - partial resection followed by reconstruction by placing a patch, type 3 - circumferential venous resection followed by anastomosis, and type 4 circumferential venous resection followed by graft interposition (19). One of the most reluctant studies conducted on this issue was published by Zhu et al. (14). The study was conducted on 52 patients diagnosed with pancreatic head cancer invading the superior mesenteric/portal vein. According to the site and extent of invasion, the authors classified the cases into four categories: cases included in the first two types presented limited local invasion that was suitable for venous resection followed by reconstruction without graft, while cases 
included in the third and fourth categories referred to larger areas of venous invasion in which grafts were needed for further reconstruction. Therefore, cases included in the first type presented lateral, limited invasion of the superior mesenteric vein, suitable for partial venous excision and direct closure of the defect, while those included in the second type presented a more extended but limited to the superior mesenteric vein invasion and were submitted to segmental resection followed by an end-to-end anastomosis. In the meantime, cases included in the third category reported a more extended invasion at the level of the superior mesenteric vein and the portal vein and necessitated graft interposition, which was anastomosed with the portal vein cranially and with the superior mesenteric vein caudally. In addition, the mesenterico-lienal confluent was laterally implanted at the level of the graft. Indubitable, the most complex reconstruction was needed in patients included in the fourth category, in which the tumoral process involved the whole length of the superior mesenteric vein and was extended cranially at the level of the portal vein; in these cases, the graft was anastomosed cranially to the remaining portal vein/portal branches and distally to the first jejunal veins, while the mesenterico-lienal confluent was implanted laterally at the level of the left side of the graft. In the study conducted by Zhu et al., there were 11 cases submitted to type I of the procedure, 15 cases submitted to type II, 18 cases submitted to type III, and only 8 cases submitted to type IV of resection and reconstruction. According to this study, patients submitted to types III and IV of resection and reconstruction reported a longer operative time, a higher amount of blood loss, a larger diameter of the tumor and a higher degree of tunica intima involvement. The number of resected and respectively positive lymph nodes were similar between the four subgroups of patients; the overall graft patency rate was of $98 \%$. As expected, cases included in the first group reported a significantly longer overall median survival time (18 months) when compared to cases included in the fourth group (in which the overall median survival time was only 10 months) (14). When it comes to the necessity of splenic vein reimplantation, different points of view have been proposed so far; therefore, while certain authors consider that after extended venous resections the splenic vein can be safely ligated, an adequate collateral circulation being expected, others consider that the risk of splenic infraction or gastric varices development is too high and therefore, they recommend to avoid this maneuver and to re-anastomose it at the level of the graft (20-24).

\section{Conclusion}

Extended venous resections have been proven to be safe and efficient in borderline resectable pancreatic cancer; depending on the location and extent of the involved segments, various types of resections and reconstructions have been proposed. However, the technique of vascular reconstruction should be carefully chosen according to the local anatomical particularities of each case.

\section{Conflicts of Interest}

The Authors have no conflicts of interest to declare in relation to this study.

\section{Authors' Contributions}

$\mathrm{NB}, \mathrm{CD}, \mathrm{OS}, \mathrm{IC}$ - preoperatively investigated the patient, VV, CS, $\mathrm{MD}, \mathrm{CB}$ - performed literature review, FF, FG, DR, IB - prepared the draft of the manuscript, $\mathrm{VB}$ - performed the surgical procedure.

\section{References}

1 Ferlay J, Shin HR, Bray F, Forman D, Mathers C and Parkin DM: Estimates of worldwide burden of cancer in 2008: GLOBOCAN 2008. Int J Cancer 127(12): 2893-2917, 2010. PMID: 21351269. DOI: 10.1002/ijc.25516

2 Jemal A, Siegel R, Ward E, Hao Y, Xu J, Murray T and Thun MJ: Cancer statistics, 2008. CA Cancer J Clin 58(2): 71-96, 2008. PMID: 18287387. DOI: 10.3322/CA.2007.0010

3 Weitz J, Kienle P, Schmidt J, Friess H and Büchler MW: Portal vein resection for advanced pancreatic head cancer. J Am Coll Surg 204(4): 712-716, 2007. PMID: 17382233. DOI: 10.1016/j.jamcollsurg.2007.01.004

4 Büchler MW, Wagner M, Schmied BM, Uhl W, Friess H and Z'graggen $\mathrm{K}$ : Changes in morbidity after pancreatic resection: toward the end of completion pancreatectomy. Arch Surg 138(12): 1310-4; discussion 1315, 2003. PMID: 14662530. DOI: 10.1001/archsurg.138.12.1310

5 Richter A, Niedergethmann M, Sturm JW, Lorenz D, Post S and Trede M: Long-term results of partial pancreaticoduodenectomy for ductal adenocarcinoma of the pancreatic head: 25-year experience. World J Surg 27(3): 324-329, 2003. PMID: 12607060. DOI: $10.1007 / \mathrm{s} 00268-002-6659-\mathrm{z}$

6 Winter JM, Cameron JL, Campbell KA, Arnold MA, Chang DC, Coleman J, Hodgin MB, Sauter PK, Hruban RH, Riall TS, Schulick RD, Choti MA, Lillemoe KD and Yeo CJ: 1423 pancreaticoduodenectomies for pancreatic cancer: A singleinstitution experience. J Gastrointest Surg 10(9): 1199-210; discussion 1210-1, 2006. PMID: 17114007. DOI: 10.1016/ j.gassur.2006.08.018

7 Tseng JF, Raut CP, Lee JE, Pisters PW, Vauthey JN, Abdalla EK, Gomez HF, Sun CC, Crane CH, Wolff RA and Evans DB: Pancreaticoduodenectomy with vascular resection: margin status and survival duration. J Gastrointest Surg 8(8): 935-49; discussion 949-50, 2004. PMID: 15585381. DOI: 10.1016/ j.gassur.2004.09.046

8 Nakao A, Kanzaki A, Fujii T, Kodera Y, Yamada S, Sugimoto H, Nomoto S, Nakamura S, Morita S and Takeda S: Correlation between radiographic classification and pathological grade of portal vein wall invasion in pancreatic head cancer. Ann Surg 255(1): 103-108, 2012. PMID: 22156923. DOI: 10.1097/ SLA.0b013e318237872e 
9 Weitz J, Koch M, Friess H and Büchler MW: Impact of volume and specialization for cancer surgery. Dig Surg 21(4): 253-261, 2004. PMID: 15308864. DOI: $10.1159 / 000080198$

10 Wang WL, Ye S, Yan S, Shen Y, Zhang M, Wu J and Zheng SS: Pancreaticoduodenectomy with portal vein/superior mesenteric vein resection for patients with pancreatic cancer with venous invasion. Hepatobiliary Pancreat Dis Int 14(4): 429-435, 2015 PMID: 26256089. DOI: 10.1016/s1499-3872(15)60400-3

11 Moore GE, Sako Y and Thomas LB: Radical pancreatoduodenectomy with resection and reanastomosis of the superior mesenteric vein. Surgery 30(3): 550-553, 1951. PMID: 14866700.

12 Fortner JG: Regional resection of cancer of the pancreas: a new surgical approach. Surgery 73(2): 307-320, 1973. PMID: 4265314.

13 Allema JH, Reinders ME, van Gulik TM, van Leeuwen DJ, de Wit LT, Verbeek PC and Gouma DJ: Portal vein resection in patients undergoing pancreatoduodenectomy for carcinoma of the pancreatic head. Br J Surg 81(11): 1642-1646, 1994. PMID: 7827892. DOI: $10.1002 /$ bjs.1800811126

14 Zhu J, Li X, Kou J, Ma J, Li L, Fan H, Lang R and He Q: Proposed Chaoyang vascular classification for superior mesentericportal vein invasion, resection, and reconstruction in patients with pancreatic head cancer during pancreaticoduodenectomy - A retrospective cohort study. Int J Surg 53: 292-297, 2018. PMID: 29654962. DOI: 10.1016/j.ijsu.2018.04.011

15 Brasoveanu V, Anghel C, Barbu I, Pautov M, Ionescu MI, Motthor M, Balescu I, Dima $S$ and Bacalbasa N: Pancreatoduodenectomy en bloc with portal and superior mesenteric artery resection-a case report and literature review. Anticancer Res 35(3): 1613-1618, 2015. PMID: 25750318.

16 Braşoveanu V, Dumitraşcu T, Bacalbaşa N and Zamfir R: Splenic artery used for replaced common hepatic artery reconstruction during pancreatoduodenectomy - a case report. Chirurgia (Bucur) 104(4): 499-504, 2009. PMID: 19886062.

17 Bacalbasa N, Balescu I, Tanase A, Brezean I, Vilcu M and Brasoveanu V: Successful resection of a non-functional paraganglioma with celiac trunk invasion followed by common hepatic artery reimplantation - A case report and literature review. In Vivo 32(4): 911-914, 2018. PMID: 29936479. DOI: 10.21873/invivo.11328

18 Bacalbasa N, Balescu I, Tanase A, Pautov M, Brezean I, Vilcu $\mathrm{M}$ and Brasoveanu V: Spleno-pancreatectomy en bloc with parcelar gastrectomy for splenic artery aneurysm - A case report and literature review. In Vivo 32(4): 915-919, 2018. PMID: 29936480. DOI: 10.21873/invivo.11329
19 Bockhorn M, Uzunoglu FG, Adham M, Imrie C, Milicevic M, Sandberg AA, Asbun HJ, Bassi C, Büchler M, Charnley RM, Conlon K, Cruz LF, Dervenis C, Fingerhutt A, Friess H, Gouma DJ, Hartwig W, Lillemoe KD, Montorsi M, Neoptolemos JP, Shrikhande SV, Takaori K, Traverso W, Vashist YK, Vollmer C, Yeo CJ, Izbicki JR and International Study Group of Pancreatic Surgery: Borderline resectable pancreatic cancer: a consensus statement by the International Study Group of Pancreatic Surgery (ISGPS). Surgery 155(6): 977-988, 2014. PMID: 24856119. DOI: 10.1016/j.surg.2014.02.001

20 Lv GY, Wang GY, Jiang C, Ji B, Wang YC, Qiu W, Sun XD and Liu YH: Laparoscopic spleen-preserving distal pancreatectomy with or without splenic vessel conservation: a retrospective study of 20 cases. Hepatogastroenterology 60(127): 1785-1788, 2013. PMID: 24624457.

21 Butturini G, Inama M, Malleo G, Manfredi R, Melotti GL, Piccoli M, Perandini S, Pederzoli P and Bassi C: Perioperative and long-term results of laparoscopic spleen-preserving distal pancreatectomy with or without splenic vessels conservation: a retrospective analysis. J Surg Oncol 105(4): 387-392, 2012. PMID: 22025322. DOI: 10.1002/jso.22117

22 Petrucciani N, Debs T, Rosso E, Addeo P, Antolino L, Magistri P, Gugenheim J, Ben Amor I, Aurello P, D’Angelo F, Nigri G, Di Benedetto F, Iannelli A and Ramacciato G: Left-sided portal hypertension after pancreatoduodenectomy with resection of the portal/superior mesenteric vein confluence. Results of a systematic review. Surgery 168(3): 434-439, 2020. PMID: 32600882. DOI: $10.1016 /$ j.surg .2020.04.030

23 Addeo P, De Mathelin P, Averous G, Tambou-Nguipi M, Terrone A, Schaaf C, Dufour P and Bachellier P: The left splenorenal venous shunt decreases clinical signs of sinistral portal hypertension associated with splenic vein ligation during pancreaticoduodenectomy with venous resection. Surgery 168(2): 267-273, 2020. PMID: 32536489. DOI: 10.1016/j.surg.2020.04.033

24 Chirletti P, Farelli F, Benucci C, Schiratti M, Prezioso G, Caronna $\mathrm{R}$, corona $\mathrm{M}$ and Sacco L: Severe intestinal bleeding due to leftsided portal hypertension after pancreatoduodenectomy with portal resection and splenic vein ligation. MOJ Tumor Res 1(1): 1-3, 2017. DOI: 10.15406/mojtr.2017.01.00001

Received May 9, 2021

Revised May 31, 2021

Accepted June 1, 2021 